\title{
LA-UR-97- -3415
}

Approved for public release;

distribution is unlimited

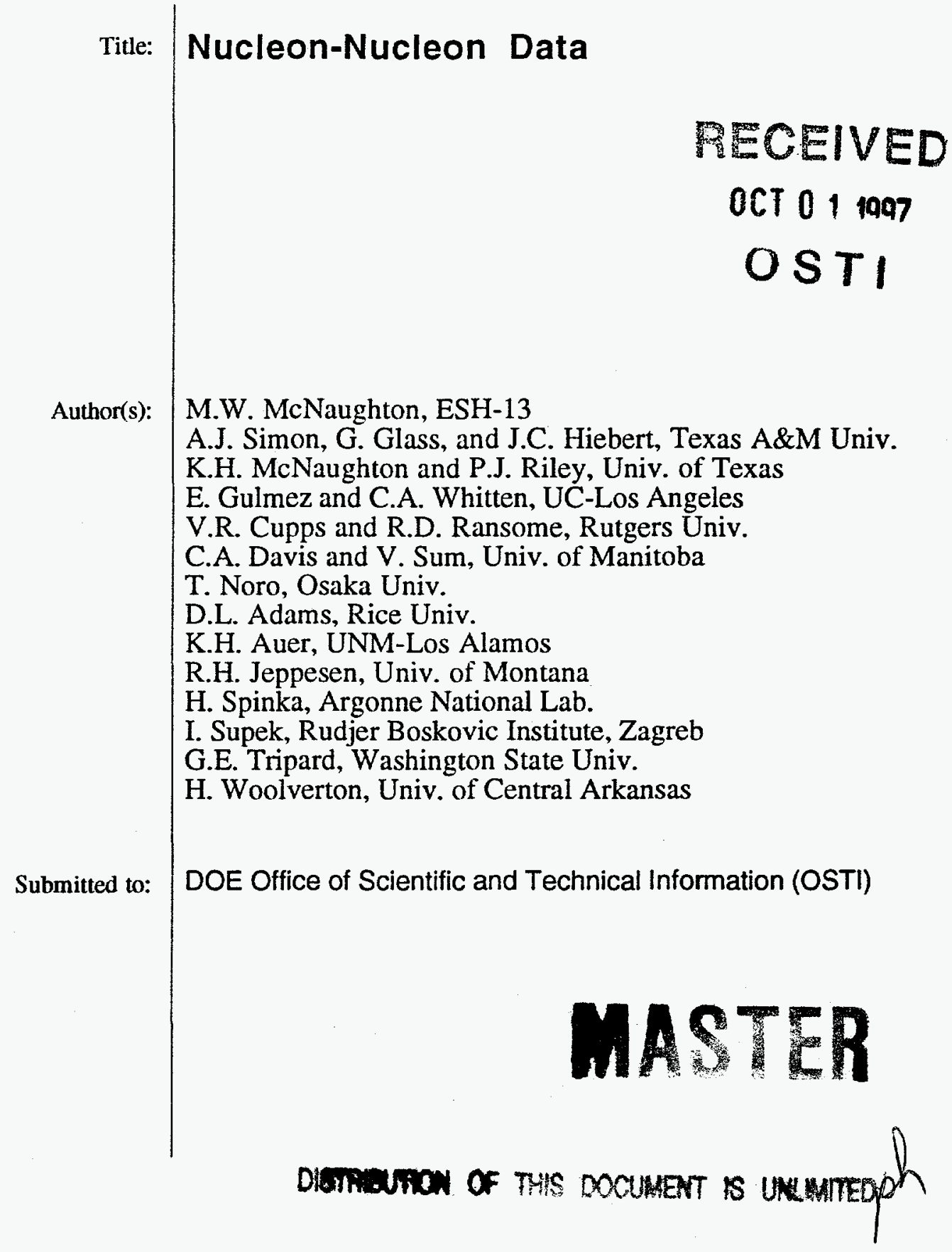

\section{Los Alamos}

NATIONAL LABORATORY

Los Alamos National Laboratory, an affirmative action/equal opportunity employer, is operated by the University of California for the U.S. Department of Energy under contract W-7405-ENG-36. By acceptance of this article, the publisher recognizes that the U.S. Government retains a nonexciusive, royalty-iree license to publish or reproduce the published torm of this contribution, or to allow others to do so, for U.S. Government purposes. The Los Alamos National Laboratory requests that the publisher identify this article as work performed under the auspices of the U.S. Department of Energy. Los Alamos National Laboratory strongly supports academic freedom and a researcher's right to publish; therefore, the Laboratory as an institution does not endorse the viewpoint of a publication or guarantee its technical correctness. 


\section{DISCLAMMER}

Portions of this document may be illegible in electronic image products. Images are produced from the best available original document. 


\section{DISCLAIMER}

This report was prepared as an account of work sponsored by an agency of the United States Government. Neither the United States Government nor any agency thereof, noi any of their employees, make any warranty, express or implied, or assumes any legal liability or responsibility for the accuracy, completeness, or usefulness of any information, apparatus, product, or process disclosed, or represents that its use would not infringe privately owned rights. Reference herein to any specific commercial product, process, or service by trade name, trademark, manufacturer, or otherwise does not necessarily constitute or imply its endorsement, recommendation, or favoring by the United States Government or any agency thereof. The views and opinions of authors expressed herein do not necessarily state or reflect those of the United States Government or any agency thereof. 


\title{
Nucleon-Nucleon Data
}

\author{
M.W. McNaughton* \\ Los Alamos National Laboratory \\ A.J Simon, G. Glass, and J.C. Hiebert \\ Texas A\&M University \\ K.H. McNaughton and P.J. Riley \\ University of Texas \\ E. Gulmez and C.A. Whitten \\ University of California at Los Angeles \\ V.R. Cupps and R.D. Ransome \\ Rutgers University \\ C.A. Davis and V. Sum \\ University of Manitoba \\ T. Noro \\ Osaka University \\ D.L. Adams \\ Rice University \\ K.H. Auer \\ University of New Mexico at Los Alamos \\ R.H. Jeppesen \\ University of Montana \\ H. Spinka \\ Argonne National Laboratory \\ I. Supek \\ Rudjer Boskovic Institute, Zagreb \\ G.E. Tripard \\ Washington State University \\ H. Woolverton \\ University of Central Arkansas
}

\begin{abstract}
This is the final report of a one-year, Laboratory Directed Research and Development (LDRD) project at Los Alamos National Laboratory (LANL). This work completes the determination of the nucleon-nucleon (NN) scattering amplitudes from 485 to $800 \mathrm{MeV}$. The data set is completed by the publication of two papers. These new data have a precision better than $1 \%$ and an absolute accuracy about $1 \%$, which is generally an order of magnitude better than previous data. The NN data set is now complete and consistent. The set of spin-dependent $\mathrm{NN}$-scattering amplitudes is now well determined up to $800 \mathrm{MeV}$.
\end{abstract}

\footnotetext{
- Principal Investigator, E-mail: mcnaughton@lanl.gov
} 


\section{Background and Research Objectives}

Nucleon-nucleon (NN) data are fundamental to nuclear physics. A precise knowledge of the NN-scattering amplitudes is an essential prerequisite for microscopic models of the nucleus. In addition, $\mathrm{NN}$ data are used as a fundamental test of the strong interaction, and as a basic standard for the normalization of nucleon-nucleus data.

A complete description of $\mathrm{NN}$ scattering at a particular angle and energy requires 10 complex scattering amplitudes. These may be derived directly from 20 independent measurements or deduced from a smaller number of measurements via a phase-shift analysis supplemented by dispersion relations.

The phase-shift approach works well at lower energies, especially near or below the pion-production threshold, where the number of orbital angular momentum states is small and the inelastic channels are negligible or confined to one resonant state. At higher energies ( $>500 \mathrm{MeV}$ ) the number of angular momentum states becomes comparable with the number of amplitudes, and the inelasticity becomes more complicated. The problem of extracting a unique solution is compounded by incorrect or inaccurate data. Consequently, more than 20 independent experiments are required.

\section{Importance to LANL's Science and Technology Base and National R\&D Needs}

Neutron scattering involves both isospin-zero and isospin-one states. The isospinone amplitudes are most easily measured via the proton-proton (pp) interaction, while the isospin-zero amplitudes must be obtained from neutron-proton (np) scattering. Thus, both $\mathrm{pp}$ and $\mathrm{np}$ data are essential for a complete understanding. The work reported here completes the measurement of both pp and np scattering.

\section{Scientific Approach and Accomplishments}

The publication "Absolute pp-Elastic Cross Sections from 492 to $793 \mathrm{MeV}$ " describes the world's most accurate measurement of pp scattering, with absolute accuracy better than $1 \%{ }^{1}$ These data have stabilized the phase-shift solutions, especially near 650 $\mathrm{MeV}$ where the opening of several inelastic channels gives rise to a resonance-like, 
energy-dependent structure in several observables. These data complete the set of $15 \mathrm{pp}$ observables and lead to a unique set of isospin-one phase shifts and amplitudes.

The publication "np-Elastic Analyzing Power at 485 and $788 \mathrm{MeV}$ " describes the world's most accurate measurement of np scattering, with point-to-point precision of 0.004 and absolute accuracy of $2 \% .^{2}$ These new data resolve a long-standing controversy on the absolute normalization of the polarized neutron beams and clarify the isospin-zero inelasticity, showing that it is coupled to a pair of delta resonances. Since $788 \mathrm{MeV}$ is far below the mean energy of a pair of deltas $(1380 \mathrm{MeV})$, this implies that the isospin-zero inelasticity is small near the maximum energy of the Los Alamos Neutron Science Center (LANSCE) accelerator.

\section{Publications}

1. Simon, A.J., et al., "Absolute pp-Elastic Cross Sections from 492 to $793 \mathrm{MeV}$," Phys. Rev. C 53, 30 (1996).

2. McNaughton, M.W., et al., "np-Elastic Analyzing Power at 485 and $788 \mathrm{MeV}$," Phys. Rev. C 53, 1092 (1996). 


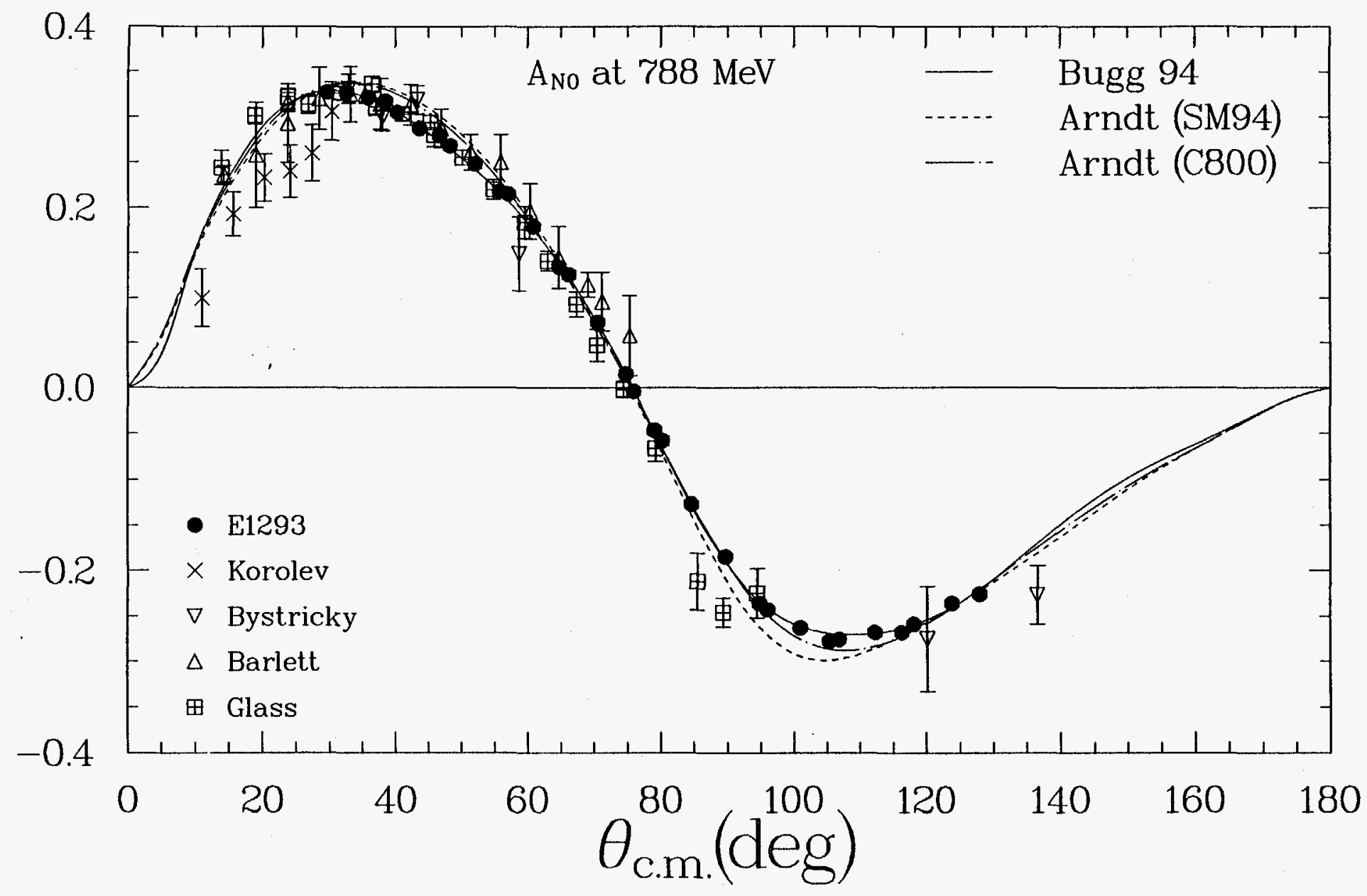

Figure 1. np-elastic analyzing power at $788 \mathrm{MeV}$, in comparison with the phase-shift fits of Arndt and Bugg, and previous data. 


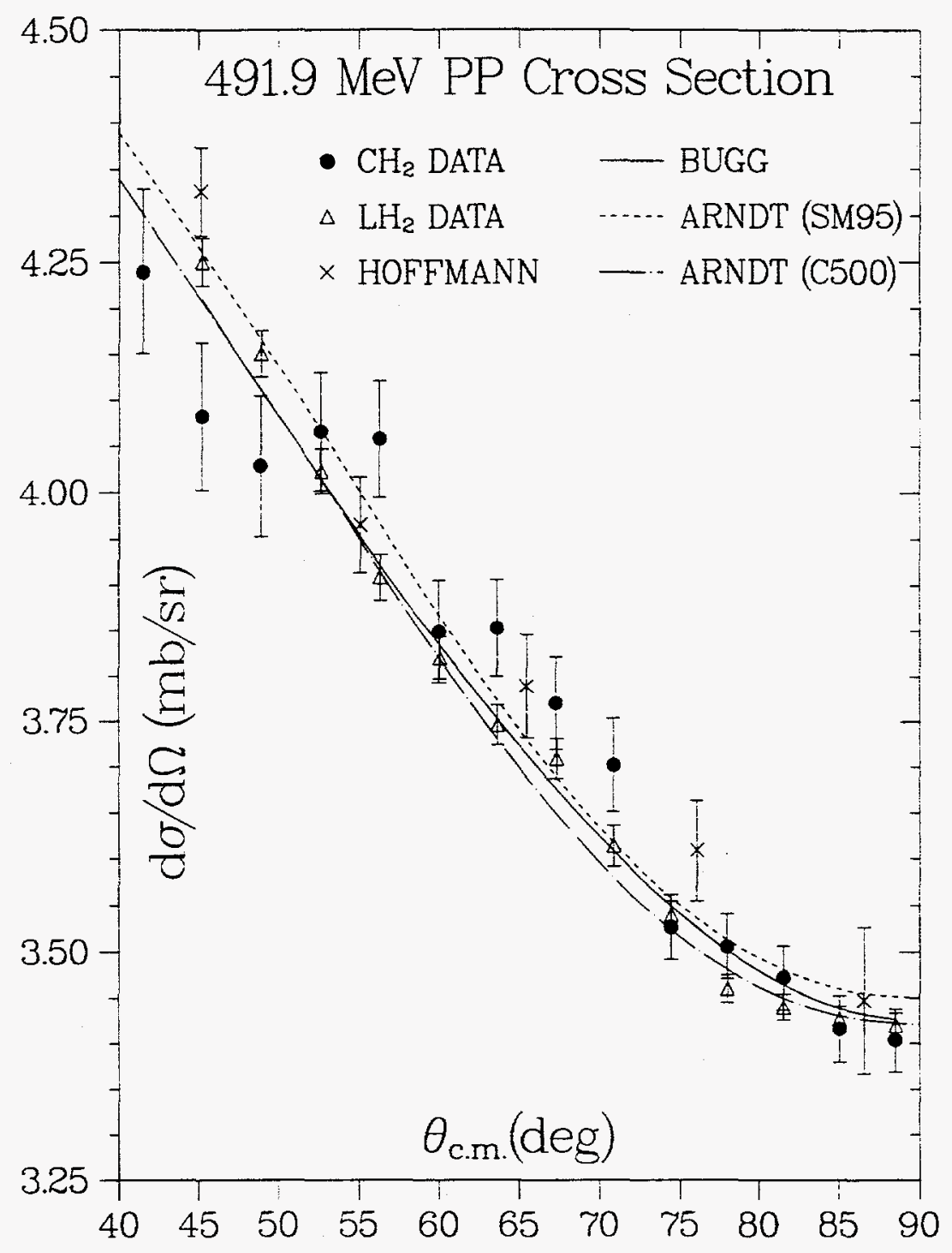

Figure 2. pp-elastic differential cross section at $492 \mathrm{MeV}$, in comparison with the phaseshift fits of Arndt and Bugg, and previous data. 